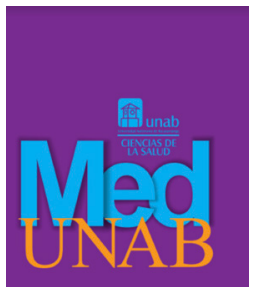

REVISTA DE LA FACULTAD

DE CIENCIAS DE LA SALUD

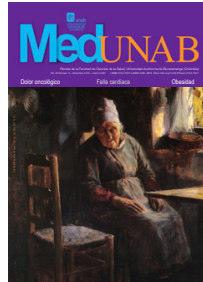

\title{
Intentos para perder peso en una población con sobrepeso y obesidad referida a un centro de endocrinología en Colombia
}

Attempts to lose weight in an overweight and obese population referred to an endocrinology center in Colombia

Tentativas de perda de peso de uma população com sobrepeso e obesidade encaminhada para um centro de endocrinologia na Colômbia

Edwin Antonio Wandurraga, MD., Esp., MSc. ${ }^{1}$ (D), Lisseth Fernanda Marín Carrillo, MD., Esp. ${ }^{2} \mathbb{D}$, María Alejandra Ardila Gutiérrez, Est. ${ }^{3}$, Sergio Eduardo Serrano-Gómez, MD. MSc. ${ }^{4}$ (D)

1. Médico, Especialista en Medicina Interna, Especialista en Endocrinología, Magíster en Oncología Molecular, Universidad Autónoma de Bucaramanga, Endocrinólogos del Oriente (ENDORIENTE), Bucaramanga, Santander, Colombia.

2. Médico, Especialista en Medicina Interna, Especialista en Endocrinología, Centro Médico Carlos Ardila-Lülle, Fundación Cardiovascular de Colombia, Bucaramanga, Santander, Colombia.

3. Estudiante de Medicina, Universidad Autónoma de Bucaramanga, Bucaramanga, Santander, Colombia.

4. Médico, Magíster en Epidemiología. Grupo de Investigaciones Clínicas de Bucaramanga, Universidad Autónoma de Bucaramanga, Bucaramanga, Santander, Colombia.

Correspondencia. Edwin Antonio Wandurraga. Especialista en Medicina Interna, Especialista en Endocrinología, Magíster en Oncología Molecular, Universidad Autónoma de Bucaramanga, Endocrinólogos del Oriente (ENDORIENTE), Calle 158 No. 20 - 55 Consultorio 408, Floridablanca, Santander, Colombia. Email. edwinwandurraga@gmail.com

\section{INFORMACIÓN DEL ARTÍCULO:}

Artículo recibido: 19 de marzo de 2019

Artículo aceptado: 09 de octubre de 2019

doi: https://doi.org/10.29375/01237047.3569

Cómo citar. Wandurraga EA, Marín Carrillo LF, Ardila Gutiérrez MA, Serrano-Gómez SE. Intentos para perder peso en una población con sobrepeso y obesidad referida a un centro de endocrinología en Colombia. MedUNAB. 2019:22(3): 314-321. doi: 10.29375/01237047.3569 


\section{RESUMEN}

Introducción. El exceso de peso es una condición prevalente en Colombia. Esto conlleva a realizar múltiples intentos para perder peso, muchos autodirigidos y con riesgos, siendo un motivo de consulta frecuente en atención médica primaria y especializada.

Metodología. Estudio de corte transversal con datos secundarios de la consulta de endocrinología de pacientes que consultaron por percepción de aumento de peso. Se indagó por 18 métodos convencionales y populares para perder peso, su duración, peso perdido y posterior re ganancia.

Resultados. Se incluyeron 100 personas, $79 \%$ mujeres, con un promedio de edad de 41.1 años, índice de masa corporal de $32.9 \pm 4.6 \mathrm{~kg} / \mathrm{m} 2$ y perímetro abdominal de $102.7 \pm 12.5 \mathrm{~cm}$. En promedio se registraron entre 4 y 5 intentos para perder peso por persona antes de consultar al endocrinólogo, con una mediana de historia de exceso de peso de 10 años. Todos los intentos lograron alguna pérdida con posterior reganancia del total del peso perdido, excepto liraglutida. No se encontró asociación significativa entre variables antropométricas y el número de intentos para perder peso.

Discusión. Los intentos de pérdida de peso más empleados por la población evaluadas son los que no están aprobados o carecen de evidencia científica robusta.

Conclusiones. Los pacientes con sobrepeso y obesidad realizan múltiples intentos fallidos para perder peso antes de consultar al médico especialista. La reganancia es muy frecuente, independientemente del tipo de intento.

Palabras clave:

Obesidad; Sobrepeso; Pérdida de peso; Fármacos Antiobesidad; Liraglutida.

\section{ABSTRACT}

Introduction. Excess weight is a prevailing condition in Colombia. This leads to many weight loss attempts, many self-managed and with risks, being a frequent reason for consulting primary and specialized healthcare.

Methodology. Cross-sectional study with secondary data from the endocrinology consultation of patients who made the appointment due to a perceived increase in weight. Eighteen conventional and popular ways of losing weight, their duration, the weight lost and the subsequent regained weight were investigated.

Results. One hundred people were included, $79 \%$ women with an average age of 41.1 years, a body mass index of $32.9 \pm 4.6 \mathrm{~kg} / \mathrm{m} 2$ and a waist circumference of 102.7 $\pm 12.5 \mathrm{~cm}$. Each person reported an average of four to five attempts to lose weight before consulting the endocrinologist, with a median history of being overweight of ten years. All of the attempts achieved some weight loss with subsequent regain of the total weight lost, except when using liraglutide. A significant association was not found between the anthropometric variables and the number of weight loss attempts.

Discussion. The weight loss methods most used by the assessed population are ones that are not approved or that lack strong scientific evidence.

Conclusions. Overweight or obese patients make multiple failed attempts to lose weight before consulting a specialist physician. Regain of the lost weight is frequent, regardless of the method used.

Keywords:

Obesity; overweight; weight loss; anti-obesity agents; liraglutide.

\section{RESUMO}

Introdução. Excesso de peso é uma condição prevalecente na Colômbia. Isso leva a várias tentativas de perda de peso, muitas auto-dirigidas e de risco, sendo motivo de consultas frequentes em atendimento médico primário e especializado. 
Metodologia. Estudo transversal com dados secundários da consulta de endocrinologia de pacientes que consultaram para percepção do ganho de peso. Foram investigados 18 métodos convencionais e populares para perder peso, sua duração, peso perdido e subsequente reganho.

Resultados. Foram incluídas 100 pessoas, 79\% mulheres, com idade média de 41,1 anos, índice de massa corporal de $32,9 \pm 4,6 \mathrm{~kg} / \mathrm{m} 2$ e perímetro abdominal de $102,7 \pm 12,5 \mathrm{~cm}$. Em média, foram registradas entre quatro e cinco tentativas de perda de peso por pessoa antes de consultar o endocrinologista, com uma mediana de história de excesso de peso de 10 anos. Todas as tentativas alcançaram alguma perda com subsequente re-ganancia do peso total perdido, exceto o liraglutida. Não foi encontrada associação significativa entre as variáveis antropométricas e o número de tentativas de perda de peso.

Discussão. As tentativas de perda de peso mais utilizadas pela população avaliada são aquelas que não são aprovadas ou não possuem evidências científicas robustas.

Conclusões. Pacientes com sobrepeso e obesos fazem várias tentativas fracassadas de perder peso antes de consultar o especialista. A re-ganancia de peso é frequente, independentemente do tipo de tentativa.

Palavras-chave:

Obesidade; Sobrepeso; Perda de peso; Fármacos antiobesidade; Liraglutida.

\section{Introducción}

La obesidad es un problema de salud pública a nivel mundial. La Organización Mundial de la Salud (OMS) estima que para 2016 la prevalencia de obesidad había triplicado la registrada en 1975, reportando un $13 \%$ en la edad adulta: $11 \%$ en hombres y $15 \%$ en mujeres (1), con los conocidos efectos deletéreos para la salud humana en relación con la diabetes, las enfermedades cardiovasculares y el cáncer (2). El panorama es poco alentador; se estima que, si las tendencias en aumento de peso continúan igual, la probabilidad de lograr metas globales en torno a obesidad será virtualmente de cero, con una prevalencia global superior al $21 \%$ en mujeres $y$ al $18 \%$ en hombres para el año 2025. (3)

Datos estadounidenses reportan una prevalencia de obesidad del $40 \%$ en adultos (4), así mismo, se conoce que el $43.6 \%$ y el $34.4 \%$ de las mujeres realizan algún intento para perder o mantener el peso respectivamente (5). Un informe publicado por la encuesta nacional de salud americana (NHANES) reveló que el sexo femenino, los individuos menores de 60 años, un alto ingreso económico, y el estatus de exceso de peso, fueron los factores asociados con un mayor número de intentos para perder peso (3). De la misma manera, en población joven americana y latina, el tamaño corporal grande y la autopercepción negativa del cuerpo incentiva a la búsqueda activa de la pérdida de peso (6).

En Colombia, el exceso de peso es una condición prevalente. En la encuesta nacional de salud (ENSIN) en su edición 2015, se reportó que el $37.7 \%$ de los jóvenes $\mathrm{y}$ adultos tienen sobrepeso y el $18.7 \%$ tienen obesidad, existiendo disparidad de género $(22.4 \%$ de las mujeres tienen obesidad frente al $14.4 \%$ de los hombres). Esta pandemia conlleva, sea por motivos cosméticos o de salud, a realizar múltiples intentos ortodoxos y alternativos, muchos de ellos autodirigidos y con riesgos, siendo un motivo de consulta o comorbilidad frecuente en atención médica primaria y especializada (7).

Por otra parte, la ganancia de peso y la obesidad tienen una fisiopatología compleja que involucra mecanismos genéticos, epigenéticos, un ambiente obesogénico, preferencias culturales, la nutrición perinatal, y patrones de actividad física, entre otros. Dado el origen multifactorial se requieren intervenciones con diferentes blancos terapéuticos, muchos de ellos con bajas tasas de eficacia y cifras altas de reganancia (8).

Debido a lo anterior, se hace necesario plantear alternativas y planes de tratamiento profesionales que requieren, en primera medida, conocer con mayor detalle el fenómeno de los intentos para perder peso. Puesto que la información disponible al respecto en Colombia es escasa, el objetivo del presente estudio es indagar y describir las estrategias para perder peso utilizadas por los pacientes que consultan a un servicio de endocrinología de referencia.

\section{Metodología}

Se realizó un estudio de corte transversal con datos anonimizados de la consulta de endocrinología de pacientes, cuyo motivo de consulta fue la percepción de aumento de peso, entre junio y diciembre del 2017. 
Se incluyeron variables demográficas como el sexo, la edad y la ciudad de procedencia, la edad de inicio de ganancia de peso y ganancia de peso aproximada desde entonces. Adicionalmente se interrogó el uso previo de 18 métodos convencionales y populares para perder peso, su duración, peso perdido y posterior reganancia presentada durante la intervención $o$ hasta 6 meses después de suspendida. Finalmente, se registró el número de valoraciones por nutricionista y la percepción del paciente acerca de la valoración. Los pacientes con obesidad por síndrome de Cushing, antecedente de cirugía bariátrica y las mujeres embarazadas fueron excluidos.

Los datos de interés consignados en la historia clínica durante la consulta fueron registrados en una base de datos en excel y, posteriormente, exportados al programa estadístico de Stata.

El estudio se considera de riesgo mínimo y se fundamenta en los principios generales de ética en

\begin{tabular}{lllll} 
Intento & $\mathbf{n}$ & $\begin{array}{l}\text { Duración } \\
\text { (meses) }\end{array}$ & $\begin{array}{l}\text { Pérdida de } \\
\text { Peso }(\mathbf{K g})\end{array}$ & $\begin{array}{l}\text { Reganancia } \\
\text { de Peso (Kg)* }\end{array}$ \\
\hline Té verde & 52 & $8.4 \pm 2.6$ & $4.8 \pm 0.9$ & $5.3 \pm 1$ \\
\hline Frutaplanta & 5 & $3.4 \pm 2.1$ & $6 \pm 0.5$ & $6.5 \pm 0.5$ \\
\hline Flor de Jamaica & 34 & $5.7 \pm 1.5$ & $3 \pm 1.1$ & $2.6 \pm 1.2$ \\
\hline Masajes reductores & 39 & $16.2 \pm 7.4$ & $5.2 \pm 1.0$ & $8.1 \pm 2.1$ \\
\hline $\begin{array}{l}\text { Auriculoterapia y } \\
\text { dieta Atkins }\end{array}$ & 55 & $4.4 \pm 0.5$ & $8.1 \pm 0.8$ & $8.8 \pm 1$ \\
\hline Hidrolipoclasia & 4 & $2.3 \pm 0.8$ & $8.5 \pm 2.5$ & $7.5 \pm 3.5$ \\
\hline Mesoterapia & 13 & $6 \pm 1.8$ & $8.7 \pm 3.8$ & $11.2 \pm 5.1$ \\
\hline Liposucción & 12 & $\mathrm{NA}$ & $9 \pm 2$ & $11 \pm 2.3 * *$ \\
\hline Abdominoplastia & 5 & $\mathrm{NA}$ & $13 \pm 4$ & $12.5 \pm 2.5 * *$ \\
\hline Malla lingual & 3 & $1.5 \pm 0.2$ & $11 \pm 4.3$ & $11 \pm 4.3$ \\
\hline Yesoterapia & 3 & $0.5 \pm 0.2$ & $1.5 \pm 0.5$ & 2 \\
\hline Herbalife ${ }^{\circledR}$ & 32 & $4.5 \pm 0.8$ & $6.6 \pm 1$ & $9 \pm 2.1$ \\
\hline Orlistat ${ }^{\circledR}$ & 23 & $3.1 \pm 0.6$ & $3.3 \pm 0.3$ & $3.4 \pm 0.4$ \\
\hline Sibutramina & 13 & $2.5 \pm 0.6$ & $7 \pm 1.2$ & $9 \pm 1$ \\
\hline Liraglutida & 11 & $6.1 \pm 2$ & $7.5 \pm 2.4$ & 3 \\
\hline Ultra ZX ${ }^{\circledR}$ & 8 & $4.5 \pm 3.2$ & $5.6 \pm 1.4$ & 8 \\
\hline Zero Xtreme ${ }^{\circledR}$ & & & & $13.8 \pm 6.1 * *$ \\
\hline Lipoblue ${ }^{\circledR}$ & 7 & 1 & $3.2 \pm 0.8$ & $5.5 \pm 1.7$ \\
\hline Gimnasio & 48 & $25.4 \pm 10.4$ & $12.4 \pm 4.4$ & \\
\hline & & & & \\
\hline
\end{tabular}

Tabla 1. Intentos para perder peso discriminados por método utilizado en 100 personas con sobrepeso u obesidad en un centro de referencia.

NA: No Aplica, Kg: Kilogramos. Los valores son presentados en promedios \pm desviación estándar.

* Reganancia de peso hasta 6 meses después de suspender el intento.

** Reganancia de peso hasta 12 meses después de suspender el intento

Fuente: Elaboración propia 
la investigación del Protocolo de Nüremberg y la Declaración de Helsinki.

\section{Análisis estadístico}

Se realizó un análisis univariado mediante medidas de tendencia central y dispersión para las variables continuas (promedio y desviación estándar para las variables con distribución normal, mediana y rango intercuartil para la distribución no normal), las variables

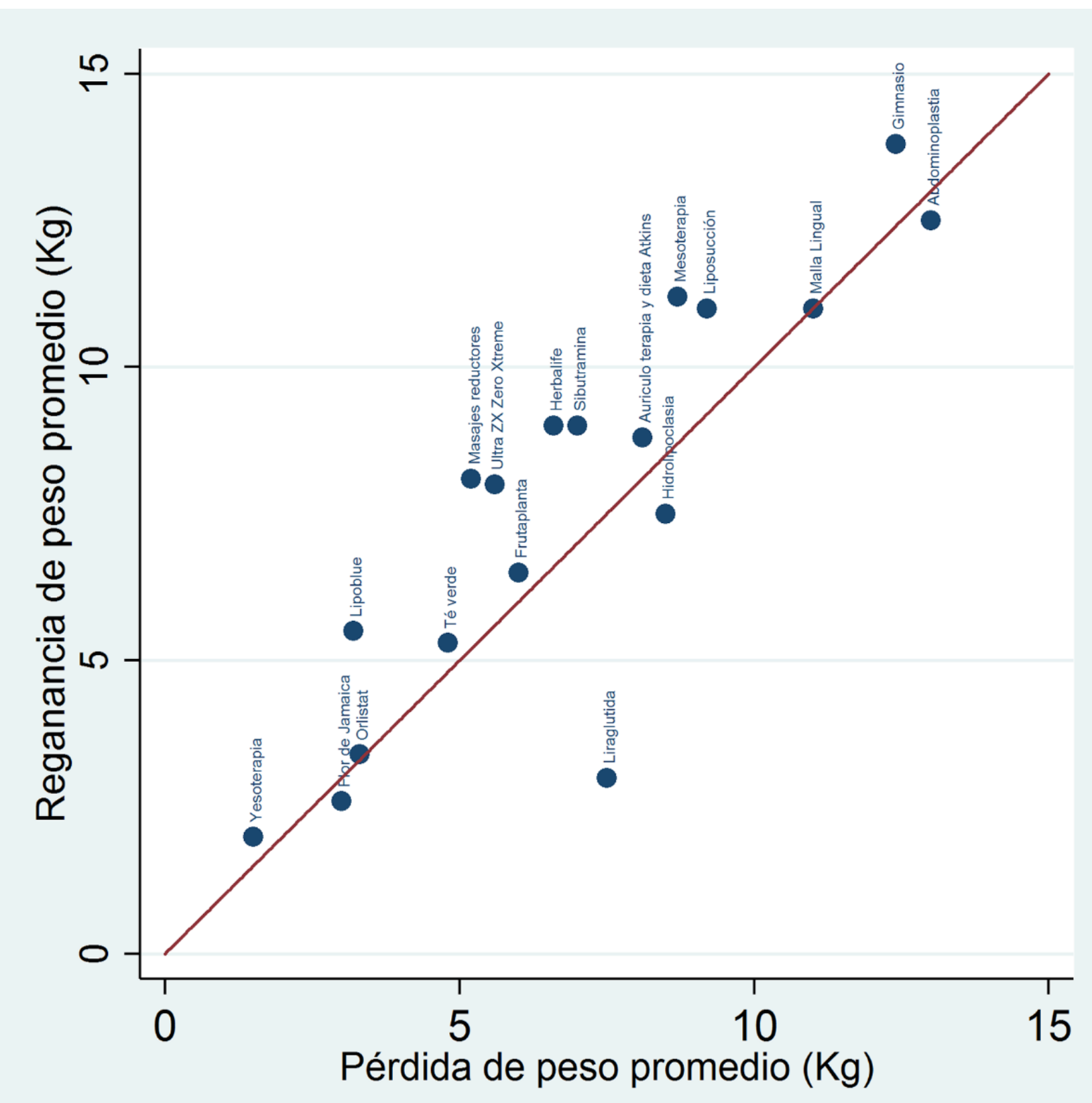

Figura 1. Comportamiento de la pérdida y reganancia de peso según el intento escogido en 100 personas con sobrepeso u obesidad en un centro de referencia.

Fuente: Elaboración propia. 
categóricas se describieron mediante frecuencias absolutas y frecuencias relativas.

Se realizó un análisis bivariado entre los métodos de pérdida de peso y las variables sociodemográficas y antropométricas, $\left(\mathrm{chi}^{2}\right.$ para las variables categóricas, y ANOVA o Kruskal Wallis para las variables continuas).

\section{Resultados}

Se incluyeron 100 participantes, 79\% mujeres, con un promedio de edad de $41.1 \pm 12.7$ años, índice de masa corporal (IMC) de $32.9 \pm 4.6 \mathrm{~kg} / \mathrm{m} 2$ y un perímetro abdominal de $102.7 \pm 12.5 \mathrm{~cm}$. El $98 \%$ de la población tenía exceso de peso (74 pacientes tenían obesidad y 24 sobrepeso). La mediana de duración de historia de exceso de peso fue 10 años con 2 o más intentos de pérdida de peso referidos en el $78 \%$ de la población y un promedio entre 4 y 5 antes de consultar al endocrinólogo. Sólo 5 pacientes refirieron no haber utilizado alguna estrategia. La ganancia de peso referida fue en promedio de $25 \pm 1.9$ $\mathrm{kg}$. Los intentos de pérdida de peso discriminados por método se describen en la tabla 1 y el comportamiento de pérdida y reganancia de peso por intento se muestran en la figura 1. No hubo diferencia en la preferencia de los intentos según el género y en el análisis bivariado no se encontró asociación con diferencia estadística entre el IMC, perímetro abdominal, sexo, edad y distribución de la grasa corporal con el número de intentos para perder peso. El 18\% de los pacientes nunca había asistido a nutricionista, los restantes, en promedio, habían asistido a valoración por nutricionista en 3 oportunidades; 19 pacientes manifestaron no tener gusto para asistir a consulta de nutrición.

\section{Discusión}

En este trabajo se evaluaron los intentos para perder peso en una población con sobrepeso y obesidad que consultó al especialista en endocrinología. Se encontró que las personas que consultan al especialista en obesidad han intentado entre 4 y 5 métodos convencionales o alternativos para perder peso. Más de la mitad de las personas que consultaron habían probado dieta Atkins (baja en carbohidratos, alta en grasas y proteínas) con o sin auriculoterapia. Un ensayo clínico demostró superioridad de esta dieta frente a un régimen convencional a 6 meses (diferencia de $4 \%$ de pérdida de peso); sin embargo, este hallazgo no fue significativo a los 12 meses (9). De manera similar, los pacientes de esta serie perdieron $8.1 \mathrm{kgs}$ con reganancia de $8.8 \mathrm{kgs}$ cuando la usaban a largo plazo o la suspendían.
Las terapias alternativas son frecuentemente utilizadas por personas con obesidad. Una serie que incluyó 31 mujeres mexicanas-americanas encontró que el $70 \%$ de las entrevistadas habían consumido té verde, $61 \%$ remedios caseros y 55\% habían recibido terapia con masajes (10). En este registro de población colombiana se encontraron resultados similares, siendo el té verde la medicina alternativa más utilizada con 8 meses en promedio de uso. Un estudio del grupo Cochrane concluyó que el consumo de té verde produce una pequeña disminución en el peso no significativa en personas con sobrepeso y obesidad (11). Por otro lado, los estudios que evalúan los masajes como terapia antiobesidad son escasos a corto plazo y siempre en combinación con acupuntura u otras terapias (12). La disminución de peso con té verde y masajes en esta población fue significativa, pero con posterior reganancia de toda la pérdida.

De manera llamativa, 8 pacientes habían tomado Zero Xtreme ${ }^{\circledR}$ y Ultra ZX®, los cuales, según alerta del INVIMA (13), contienen sibutramina (componente prohibido en Colombia desde el año 2010). En la misma situación se encuentran nutreline bluevelle ${ }^{\circledR}, 7$-day slim xtreme ${ }^{\circledR}, \mathrm{A} 1 \mathrm{slim} \AA$, bodyxtreme ${ }^{\circledR}$ y Xtreme $\mathrm{ZX}{ }^{\circledR}$. Al interrogar por el consumo de Lipo blue ${ }^{\circledR}, 7$ pacientes lo habían intentado por cuenta propia. Este producto carece de registro INVIMA por la presencia de ingredientes no declarados que pueden poner en riesgo la salud de sus consumidores. En el mismo listado aparecen Lipoblue advance $₫$, Ultra DX deluxe $₫$, G180®, G360®, Pure life cleanse ${ }^{\circledR}$ y Nitrofit ${ }^{\circ}$. Ninguno de los mencionados cuenta con registro sanitario en la actualidad y su comercialización en Colombia es ilegal (14). El consumo de este tipo de sustancias se ha observado con mayor frecuencia en población femenina, población con alto nivel de escolaridad, alto nivel de ingresos familiares, en menores de 60 años y en aquellos con diagnóstico o percepción de obesidad (15). En la presente serie no se identificó algún factor asociado al consumo de dichas sustancias.

Adicionalmente, se encontró que los pacientes tardan 10 años para consultar al especialista desde el inicio de la ganancia de peso. Si bien los intentos para perder peso son muy frecuentes en personas con obesidad, sólo el $20 \%$ en Reino Unido (16) y el $25 \%$ en Suecia (17) acude a su médico general, y únicamente el $10 \%$ de los pacientes han recibido fármacos antiobesidad formulados por doctores en Australia (18). La baja proporción de terapias dirigidas por un médico puede ser explicado por la ausencia de autopercepción de obesidad y de su connotación como enfermedad médica (19), así como por el amplio uso del internet donde los 
pacientes disponen de información, en búsqueda de "soluciones milagrosas" para su problema considerado, muchas veces, "cosmético" (18).

Como hallazgo relevante, en todas las estrategias expuestas en la tabla 1 se observó reganancia de peso hasta el nivel basal o incluso superior (efecto rebote), excepto con Liraglutida; Esta es una terapia aprobada por la FDA y el INVIMA para pacientes con IMC mayor a $30 \mathrm{Kg} / \mathrm{m} 2$, o mayor a $27 \mathrm{Kg} / \mathrm{m} 2$ con comorbilidad. En su estudio pivotal, Liraglutida mostró una reducción promedio de $8.4 \pm 7.3 \mathrm{~kg}$ a 56 semanas, esto logró una pérdida del peso corporal del $5 \%$ y $10 \%$ en el $63.2 \%$ y $33.1 \%$ de los pacientes respectivamente (21). Dentro de los mecanismos involucrados en la recuperación de peso se describen factores homeostáticos (aumento de péptidos orexígenos, disminución de los anorexígenos y de la tasa metabólica basal), ambientales (incremento en la ingesta energética) y comportamentales (activación de los sistemas de recompensa hedónica, estrés y desmotivación) (22).

Este reporte es la primera aproximación para conocer las estrategias de pérdida de peso utilizadas por los pacientes con sobrepeso y obesidad en nuestro medio y para alertar a los clínicos acerca del uso de terapias no supervisadas con riesgos para la salud. Una limitación del estudio es el potencial sesgo de recordación de datos ya que se registraron intentos previos para perder peso implementados en cualquier momento durante la historia de exceso de peso.

\section{Conclusiones}

Los pacientes con sobrepeso y obesidad tardan hasta 10 años en consultar al especialista, lo hacen luego de realizar, en promedio, entre 4 y 5 intentos para perder peso. El presente estudio demuestra la gran variedad de métodos autodirigidos que utiliza la población estudiada y enciende las alarmas acerca del uso de estos a pesar de riesgos y advertencias realizadas por entidades gubernamentales. Más aún, se observa que todos los intentos realizados presentaron reganancia del $100 \%$ de la pérdida alcanzada, exceptuando el uso de liraglutida, por lo cual el personal de salud que atiende pacientes con obesidad, debe estar familiarizado con las terapias farmacológicas aprobadas para perder peso, así como con aquellos métodos populares, teniendo en cuenta su alto uso y potenciales riesgos.

\section{Conflictos de interés y financiación}

No se recibió financiación alguna para este trabajo.

EAWS declara haber participado en advisory boards de terapias para obesidad con Novo Nordisk.

\section{Referencias}

1. Organización Mundial de la Salud. Obesidad y sobrepeso. Nota Descriptiva. Febrero 2018. [consultado octubre 01 de 2019]. Recuperado de: https://www.who.int/es/news-room/fact-sheets/ detail/obesity-and-overweight

2. Wang YC, McPherson K, Marsh T, Gortmaker SL, Brown M. Health and economic burden of the projected obesity trends in the USA and the UK. Lancet (London, England). 2011 aug;378(9793):815-825. doi:10.1016/S01406736(11)60814-3.

3. NCD Risk Factor Collaboration. Trends in adult body-mass index in 200 countries from 1975 to 2014: a pooled analysis of 1698 population-based measurement studies with 19.2 million participants. Lancet (London, England). 2016 Apr; 387(10026): 1377-96.

4. Ministerio de Salud y Protección Social, Instituto Nacional de Salud, Instituto de Prosperidad Social, Instituto Colombiano de Bienestar Familiar, Universidad Nacional de Colombia. Encuesta Nacional de Situación Nutricional de Colombia (ENSIN). 2015:1-58.

5. González-Muniesa P, Mártinez-González MA, Hu FB, Després JP, Matsuzawa Y, Loos RJF, et al. Obesity. Nat Rev Dis Primers. 2017 Jun 15;3:17034. doi: 10.1038/nrdp.2017.34.

6. Martin CB, Herrick KA, Sarafrazi N, Ogden CL. Attempts to Lose Weight Among Adults in the United States, 2013-2016. NCHS Data Brief. 2018 Jul;(313):1-8.

7. Serdula MK, Mokdad AH, Williamson DF, Galuska DA, Mendlein JM, Heath GW. Prevalence of attempting weight loss and strategies for controlling weight. JAMA 1999;282(14):1353-8.

8. Epperson AE, Song AV, Wallander JL, Markham C, Cuccaro P, Elliott MN, Schuster MA. Associations among body size, body image perceptions, and weight loss attempts among African American, Latino, and White youth: a test of a mediational model. J Pediatr Psychol. 2014 May;39(4):394404. doi: 10.1093/jpepsy/jst096.

9. Foster GD, Wyatt HR, Hill JO, McGuckin BG, Brill $\mathrm{C}$, Mohammed BS, et al. A randomized trial of a 
low-carbohydrate diet for obesity. N Engl J Med. 2003;348(21):2082-90.

10. Lindberg NM, Stevens VJ, Elder C, Funk K, Debar L. Use of alternative medicine for weight loss among Mexican-American women. J Immigr Minor Health. 2013 Oct;15(5):982-5. doi: 10.1007/s10903-0129674-7.

11. Jurgens TM, Whelan AM, Killian L, Doucette S, Kirk S, Foy E. Green tea for weight loss and weight maintenance in overweight or obese adults. Cochrane Database Syst Rev. 2012 Dec 12;12:CD008650. doi: 10.1002/14651858.CD008650.pub2.

12. He J, Zhang X, Qu Y, Huang H, Liu X, Du J, Guo S. Effect of Combined Manual Acupuncture and Massage on Body Weight and Body Mass Index Reduction in Obese and Overweight Women: A Randomized, Short-term Clinical Trial. J Acupunct Meridian Stud. 2015 Apr;8(2):61-5. 10.1016/j.jams.2014.08.001.

13. Invima [internet]. Colombia: 27-03-2017. Disponible: https://www.invima.gov.co/medicamentos-yproductos-biologicos-aler-sani/27-03-2017-invimaalerta-sobre-los-producto-publicitado-como-zeroxtreme-pdf/detail.html

14. Invima [internet]. Colombia. Disponible: https:// www.invima.gov.co/medicamentos-y-productosbiologicos-aler-sani/alerta-sanitaria-lipo-blue-pdf/ detail.html

15. Machado EC, Silveira MF, Silveira VM. Prevalence of weight-loss strategies and use of substances for weight-loss among adults: a population study. Cad Saude Publica. 2012 Aug;28(8):1439-49.

16. Evans EH, Sainsbury K, Kwasnicka D, Bolster A, Araujo-Soares V, Sniehotta FF. Support needs of patients with obesity in primary care: a practice-list survey. BMC Fam Pract. 2018; 19: 6. doi: 10.1186/ s12875-017-0703-4.
17. Zenténius E, Andersson-Assarsson JC, Carlsson LMS, Svensson PA, Larsson I. Self-Reported WeightLoss Methods and Weight Change: Ten-Year Analysis in the Swedish Obese Subjects Study Control Group. Obesity (Silver Spring). 2018 Jul;26(7):1137-1143. doi: 10.1002/oby.22200.

18. Yoong SL, Carey ML, Sanson-Fisher RW, D'Este C. A cross-sectional study assessing the self-reported weight loss strategies used by adult Australian general practice patients. BMC Fam Pract. 2012 May 30;13:48. doi: 10.1186/1471-2296-13-48.

19. Truesdale KP, Stevens J. Do the Obese Know They Are Obese? N C Med J. 2008; 69(3): 188-194.

20. Lewis S, Thomas SL, Blood RW, Castle D. Hyde J, Komesaroff PA. Im searching for solutions: why are obese individuals turning to the Internet for help and support with being fat? Health Expect. 2011;(4): 339350. doi: 10.1111/j.1369-7625.2010.00644.x.

21. Pi-Sunyer $\mathrm{X}$, Astrup A, Fujioka K, Greenway F, Halpern A, Krempf M.A et al. Randomized, Controlled Trial of $3.0 \mathrm{mg}$ of Liraglutide in weight management. N Engl J Med. 2015 Jul 2;373(1):11-22. doi: 10.1056/NEJMoa1411892

22. Greenway FL. Physiological adaptations to weight loss and factors favouring weight regain. Int J Obes (Lond). 2015 Aug;39(8):1188-96. doi: 10.1038/ ijo.2015.59. 